\title{
ALL'OMBRA DI CAPUANA: ADELAIDE BERNARDINI SCRITTRICE E DRAMMATURGA
}

\author{
IN THE SHADOW OF CAPUANA: ADELAIDE BERNARDINI, WRITER AND \\ PLAYWRIGHT
}

Dora Marchese

Università di Catania

\section{Riassunto:}

Si analizzano la figura e l'opera di Adelaide Bernardini, poetessa, narratrice, giornalista e drammaturga, ricordata in genere solo come giovane moglie del grande Luigi Capuana ma che invece fu scrittrice feconda, varia nei generi e nei temi, promotrice d'istanze favorevoli alle donne, ed infaticabile imprenditrice dell'opera sua e del marito, specie in campo teatrale dove collaborò con grandi artisti e intellettuali come Nino Martoglio, Giovanni Grasso e Angelo Musco.

\section{Parole chiave:}

Teatro siciliano, Luigi Capuana, ribellismo femminile.

\section{Abstract:}

An analysis of the figure and the works of Adelaide Bernardini - poetess, storyteller, journalist and playwright. She is usually only remembered as the young wife of the great Luigi Capuana, nevertheless she was a prolific writer who explored many genres and themes, she promoted several initiatives in favour of women and was a tireless entrepreneur for both hers and her husband's work - especially in theatre where she worked with important artists and intellectuals as Nino Martoglio, Giovanni Grasso and Angelo Musco.

\section{KeYwORDS:}

Sicilian theatre, favour of women, Luigi Capuana. 
Poetessa, narratrice, giornalista e drammaturga, Adelaide Bernardini è ricordata solo come la giovane moglie del celebre Luigi Capuana, mentre sulla sua opera, ampia e varia per generi e temi, è caduto irrimediabilmente l'oblio.

La maggior parte di coloro che hanno scritto della Bernardini non sono stati benevoli nei suoi confronti, accusandola più o meno esplicitamente di aver utilizzato il nome e la fama del marito per fare carriera, di essere, dunque, la scaltra manipolatrice di un uomo più anziano, fragile perché innamorato, e considerandola, sostanzialmente, una scrittrice di nessun talento, parassitaria, anche in questo caso, nei confronti dell'illustre consorte.

A tutto ciò si aggiunse il riverbero di negatività gettato su di lei dalle polemiche sorte con Pirandello, dai rapporti tesi con Verga, Musco, Martoglio ed altri amici e intellettuali a vario titolo legati a Capuana, ed anche dalla difficoltà insita nel carattere irruento e polemico della Bernardini che non di rado puntualizzava, sottolineava, analizzava, in lettere o articoli fluviali, le accuse e le critiche che venivano mosse a lei ed al marito per ricusarle con violenta risolutezza (ricordiamo, ad esempio, il femminile antagonismo verso Sibilla Aleramo, Jolanda, Luigi di San Giusto, Francesca Sabato Agnetta $)^{1}$.

Tuttavia la realtà, come spesso accade, è più complessa e la distanza che il tempo ha posto tra la vicenda esistenziale ed artistica della Bernardini e noi ci consente oggi un più sereno giudizio.

Adelaide Bernardini nasce a Narni, in provincia di Terni, secondo alcuni nel 1872, secondo altri nel 1876, ma vive quasi sempre in territorio catanese, dove muore il 2 novembre del 1944. Maestra elementare, dal suo paese si trasferisce giovanissima a Smirne e Costantinopoli anche a seguito della perdita di entrambi i genitori. Innamoratasi di un ufficiale dell'esercito che, condottala con sé a Roma, la seduce e poi la abbandona, Adelaide decide di uccidersi consegnando il suo epitaffio ad un biglietto lasciato nell'albergo scelto per l'atto estremo.

La notizia della giovane donna sopravvissuta al tentato suicidio fa scalpore e viene menzionata sui giornali. È allora che, come in un romanzo, entra in scena Luigi Capuana, maturo ed affermato intellettuale da tempo trasferitosi a Roma dalla Sicilia. Il quasi sessantenne Capuana, impressionato dalla vicenda, offre tramite lettera un lavoro "da segretaria, da custode di una ricca biblioteca, in una casa signorile" alla ventitreenne Bernardini (Verdirame, 2009: 39). Dopo il primo incontro, avvenuto nel 1895 nel salotto di un professore di violino, i due iniziano una frequentazione che sfocerà in una lunga convivenza e quindi nel matrimonio tenutosi il 23 aprile 1908 a

1 La prima era l'amante di Musco; le altre sono indicate con i loro pseudonimi che sono, rispettivamente, quelli di Rina Faccio, Maria Majocchi Plattis e Luisa Mancina Gervasio. 
Catania, dove Capuana insegna "Lessicografia e Stilistica" all'Università; testimone degli sposi l'amico di sempre Giovanni Verga.

Fin qui la "favola".

In realtà la vita di Adelaide non fu sempre rose e fiori. Accanto all'affetto sincero e grato nei confronti di un uomo che tanto le era legato, che la stimava e la sosteneva con tutto se stesso, che l'aveva sottratta alla morte e al disdoro, che con commosso entusiasmo la rese partecipe della sua vita, Adelaide si trovò a dovere fronteggiare anche l'ambiente lavorativo, sociale e familiare di Capuana, le sue abitudini e la sua mentalità di uomo (spesso diversa da quella dello scrittore), i tanti problemi finanziari, la notevole differenza di età, l'ostilità spesso gratuita dei tanti che gravitavano nell'orbita del Maestro e che vedevano in lei - donna - un ostacolo imprevisto e inaccettabile.

Anche perché la Bernardini non rinunciò mai alle sue aspirazioni artistiche. Anzi continuò a scrivere ${ }^{2}$ e senza dubbio vide in suo marito un valido e legittimo volano per la celebrità e il successo. Fino al 1920 circa, infatti, adoperò il doppio cognome Capuana Bernardini per firmare le sue opere e questa scelta fu quasi coralmente giudicata interessata e strumentale. Altrettanto strumentale, e non frutto di profonda e sincera convinzione, venne considerato il continuo premere di Capuana verso amici e colleghi, scrittori, giornalisti, impresari e attori, per recensire, mettere in scena, pubblicare e pubblicizzare le opere della moglie. La Bernardini appariva, dunque, un'abilissima manipolatrice che aveva sobillato e stregato Capuana, facendogli perdere lucidità e obiettività.

Questi pesanti pregiudizi, unitamente a velenose ed aspre polemiche che sorsero contro di lei (ricordiamo, ad es., il feroce attacco di Francesco Biondolillo ${ }^{3}$, ma anche la querelle con Luigi Pirandello a cui accenneremo più avanti) non hanno permesso, a mio giudizio, una valutazione serena e obiettiva dell'opera della Bernardini, scrittrice prolifera, pronta a cimentarsi in vari generi letterari, in cerca di un suo stile e di una sua autonomia poetica e intellettuale.

Pur se in modo discontinuo e con esiti non sempre felici, Adelaide Bernardini ha avuto il merito, nel febbrile e movimentato scorcio di fine '800-inizi '900, di non porsi pedissequamente nel solco delle scelte letterarie del marito ma di cercare, talvolta disordinatamente, una strada sua d'avanguardia; di avere espresso un nuovo atteggiamento all'interno della letteratura al femminile; di aver partecipato in maniera attiva, facendogli da "impresaria", alla gestione e organizzazione dell'imponente messe

2 Adelaide racconta: "La letteratura mi attirava [...] cominciai a pubblicare i primi versi, le prime novelle, i primi drammi, ne traevo notorietà e guadagni" (cfr. Verdirame, 2009: 40).

3 Francesco Biondolillo fu l'autore di un impietoso e violento libello polemico dal titolo Macellatio Capuanae Bernardinaeque del 1913. 
di impegni, contratti e rapporti di Capuana con attori, editori, traduttori, colleghi, familiari e persino creditori.

Sin da giovanissima la Bernardini compone poesie (alcune tradotte in spagnolo) e novelle (tradotte in francese e in tedesco), per poi cimentarsi con romanzi e drammi e con il giornalismo, collaborando con diverse testate: da quelle nazionali, molto note, come "Fanfulla della Domenica", "Secolo XX", "Giornale d'Italia", a quelle a diffusione territoriale, come "L'Ora", "Sicilia", “Tirso" e il catanese "Giornale dell'Isola Letterario"; da quelle destinate ad un pubblico femminile, in voga in quegli anni, come "Cordelia", "Donna”, a periodici di taglio letterario come "Nuova parola”, "Natura ed Arte", "Poesia", “Regina".

Tra le raccolte poetiche ricordiamo: Intime (1897), Nuove intime (1898), Flos animae (1900), Rime amorose (1903), Amaritudini (1911), Sottovoce (1911).

In ambito narrativo, oltre alle novelle Dopo il no (1899); Prime novelle (1899); Contessina (1899, poi 1926); Le spine delle rose (1904); La vita urge... (1908); Marionette da salotto (1920); La Signora Vita e la Signora Morte (1920), la Bernardini scrive testi per l'infanzia (Tutt'ora) e, con Luigi Capuana, il libro per ragazzi Il figlio di Scurpiddu. Nella silloge Le spine delle rose sono annunciate di prossima pubblicazione La Nemica (dramma in tre atti), La indocile preda (poesie) e Biondella (racconto per bambini). Nel volume Prime novelle si dichiarano in uscita il romanzo Mai!, La istitutrice (dramma in tre atti) e, in preparazione, Falene (versi) ed In provincia (racconto per signorine).

Sul fronte drammaturgico si annoverano: Fulvia Tei (atto unico-1897); Bufera (atto unico-1899); Rovina (atto unico-1902) ${ }^{4}$; Come l'edera (atto unico-1904); Un perché (atto unico-1905); Ammatula! (atto unico-1908- tradotto in siciliano da Luigi Capuana); Nonostante (atto unico-1909); Da cosa nasce cosa (scene-1912); Buon sangue non mente (atto unico-1915); L'integro (1917), I Ballarò (dalla novella I Bestia di Luigi Capuana) e Lu Picuraru e la picuredda, commedie, queste ultime, in tre atti tradotte in siciliano da Ludovico Capuana, nipote dello scrittore. Dell'insigne marito Adelaide ha trasposto in lingua Il Cavalier Pedagna e Quacquarà.

La Bernardini è autrice anche di brevi scritti critici: Per Salvatore Giuliano, commemorazione (1910) e L'amore e il dolore nelle poesie di G.A. Cesareo (1913) e librettista: suoi sono i testi delle romanze La parola unica (1875 circa), musica di Giuseppina Lombardo e T'amo tanto! (1880 circa), musica di Arturo Marucelli.

Tra le molte polemiche in cui viene coinvolta è significativa quella con Filippo Tommaso Marinetti, sorta a causa della mancata pubblicazione della raccolta Sottovoce

4 Il copione di Rovina reca in copertina, oltre alla firma e all'indicazione "dramma in un atto, in prosa", la data 1902 e l'annotazione: "Capuana e Verga mi hanno consigliato alcuni tagli. Li ho eseguiti con poco piacere!". E sul retro dell'ultima pagina: "Di questo lavoro sono contentissima, e sento che il tempo non muterà quest'altezzoso giudizio!... Roma 29 aprile 1902". 
presso la milanese "Casa Editrice di Poesia". Marinetti chiede alla Bernardini di cambiarne il titolo, non in sintonia con i toni virili e fragorosi del futurismo inneggianti all'urlo e al paroliberismo. La scrittrice si rifiuta e pubblica la silloge con un altro editore (Giannotta, 1911) non tralasciando, però, di premettere un'autoprefazione $A l$ lettore in cui, con toni di palese risentimento, racconta come il manoscritto, accettato in un primo tempo da Marinetti "con vivissimo piacere" perché da considerarsi "grande opera di poesia, sempre ispirata, sempre traboccante come una bella sorgente, sempre originale", fu poi da lui stesso sottoposta a "sgambetto" perché "in perfetto disaccordo" con i canoni poetici futuristi (Zappulla Muscarà, 1996: 638). E dire che Capuana, in una lettera all'amico Manzella Frontini del 1910, dunque prima della rottura con l'autore del Roi Bombance, scriveva a proposito della moglie: "se sapesse che futurista ella è e da tanto tempo avanti che si parlasse di futurismo! [...] Il suo futurismo è, soprattutto, sincerità". E aggiunge - fattore da considerarsi comunque a favore dell'autonomia della Bernardini -, che malgrado avesse cercato di farle apprezzare Fogazzaro, De Amicis "e qualche altro scrittore dello stesso genere" lei, "ribelle" ma "volenterosissima", non aveva ceduto. Sicché, conclude, "quel che ha fatto proviene unicamente dagli sforzi costanti del suo vivissimo ingegno" (Zappulla Muscarà, 1996: 441-). Secondo alcuni, in questa vicenda è da ricercarsi il motivo dell'allontanamento di Capuana dal futurismo a cui, in un primo momento, lo scrittore aveva guardato con simpatia.

Come si vede l'impegno letterario della Bernardini è stato corposo e costante, non rallentato dalle feroci critiche o anche dalla semplice indifferenza dimostratole da alcuni.

Ovviamente non sono mancate le espressioni di apprezzamento. Tra queste ricordiamo quelle di Bandini Buti, Casati, Gastaldi, Rovito, Villani, e quelle, temiamo più per cortesia che per convinzione, espresse da Verga, De Roberto, Pirandello (cfr. Zappulla Muscarà, 1987: 375; 367 e Zappulla Muscarà, 1996: 576-7).

In occasione della pubblicazione della "lirica in forma di racconto" Barca Nova del 1906, la scrittrice è definita "futurista prima del Futurismo"; La vita urge è recensita positivamente dal futurista Paolo Buzzi, e tre sonetti, poi inclusi in Sottovoce ( $L a$ consolatrice, Un maestro, Rivolta) - silloge reclamizzata nell'Enquête internationale sur le Vers libre -, sono annunciati come di "imminente pubblicazione" nel catalogo editoriale di "Poesia" dal quindicinale illustrato "La Donna" di Torino. E se per alcuni la Bernardini è stata "solo apparentemente salda nella fede sulla poetica e le scelte dell'avanguardia" (Verdirame, 2010: 268), si potrebbe però osservare che, almeno finché il suo carattere cimentoso e impulsivo non la portò ad uno strappo con editori, recensori e scrittori futuristi, la forma e lo stile da lei adoperati sono stati accolti e sanciti come appartenenti all'avanguardia da testate filomarinettiane di diffusione ampia e internazionale. 
A distanza di più di settant'anni possiamo dire che non si può condividere l'opinione dei suoi tanti detrattori. Non poche pagine sono degne di ricordo, soprattutto le prose. "Scontate le raccolte di rime, intrise di autobiografismo e affettato sentimentalismo, nonostante le velleità stilistiche di originalità e limpidezza ("io ti vorrei, mio verso/ com'onda cristallina/ fresco, fluente e terso"); elegiaca la musa prosastica; meno crepuscolari le commedie borghesi e i drammi rusticani" (Verdirame, 2010: 270-1). Nella narrativa la Bernardini mostra una "gradevole scioltezza" nel contrassegnare trame che vertono su tre temi ricorrenti: l'amore infelice, la passione irrefrenabile, l'adulterio. Quest'ultimo motivo "ridondante" è avvelenato da crucci e rimorsi nelle Prime novelle e nelle Spine delle rose, per assumere "toni espressionistici" in Marionette da salotto e comparire anche, in maniera più avvertita, in Mai! e nell' Altro dissidio. Racconto (Verdirame, 2009: 55).

Oltre ad non essersi mai arresa, e ad avere continuato ad opporsi ad una damnatio memoriae da scontare già in vita, la Bernardini ha il merito di avere cercato la sua collocazione artistica non seguendo l'indirizzo di oggettività e verismo, prima, di simbolismo e esoterismo, dopo, del marito Luigi. Al contrario Adelaide percorre una strada sua, restando coerente con se stessa persino quando è Marinetti a chiederle di modificare anche un semplice titolo.

Una figura controversa, talvolta ambivalente. Rita Verdirame, ad esempio, sulla scorta della novella Fatalità, apparsa nel febbraio 1919 sul "Giornale dell'Isola Letterario", vi ravvisa "uno stile tradizionalissimo, del tutto alieno dalle audaci scorribande dei "novatori"“ (Verdirame, 2010: 271). Anna Santoro, curatrice della nuova edizione del racconto L'altro dissidio, scritto nel 1891 e pubblicato dalla Bernardini nella raccolta $\mathrm{La}$ Signora Vita e la Signora Morte, afferma invece che l'autrice "con un linguaggio moderno, scrive delle cose che sa, degli ambienti che le sono familiari", e scrivendo opera "piccole innovazioni, sempre ponendosi con decisione dal punto di vista delle donne e soprattutto delle donne che si ribellano" (Santoro, 2000). Il suo linguaggio vuole essere chiaro ed efficace. Raccontando storie di tradimenti e vendette, di omicidi e suicidi, di matrimoni fasulli o infelici, ma anche di vita comune, la Bernardini opera un ribaltamento del focus dal maschile al femminile, sancendo l'irreversibile trasformazione che si stava compiendo in quegli anni della coscienza di una dimensione ed un'identità muliebri che s'imponessero anche in letteratura. "Andar via con l'uomo che si ama è giusto, è giusto ribellarsi ad un marito che tradisce, ad una famiglia che si disprezza, è giusto respingere un concetto di perdono che può diventare catena e umiliazione, è giusto scegliere per i propri figli un destino magari di povertà ma dignitoso. [...] se pure la figura del marito padre padrone è accettata e subita da tante donne, altre però, nella realtà prima ancora che nella finzione letteraria, si ribellano, offrendosi come presenza forte che possa essere colta e raccontata" (Santoro, 2000). 
È interessante notare come la pensava a riguardo l'affermata e altrettanto battagliera Matilde Serao, scrittrice prolifera e giornalista di successo, prima donna in Italia ad aver fondato e diretto da sola l'importante quotidiano "Il Giorno", molto ammirata dalla Bernardini che le dedica il dramma Rovina.

L'opposizione uomo/donna compare nella maggior parte dei romanzi e delle novelle della Serao e suggerisce la naturale soggezione della donna all'uomo. Questo ruolo suscita fastidio e senso di ribellione, ma la Serao sembra convinta che la legge naturale da seguire sia questa. Il suo sguardo è fatalista e disincantato. Il codice comportamentale da lei proposto si fonda sull'accettazione del ruolo domestico, sull'etica del dovere, del sacrificio e del perdono, proprio a causa della debolezza della donna, della sua precarietà fisica e psicologica: "non v'è felicità possibile per la donna, in qualunque condizione essa si trovi: né nel matrimonio, né nell'amore libero, né nell'amore illegale ("Il Giorno", 17 novembre 1907). Ancor più illuminanti le parole di risposta (21 maggio 1880) ad un articolo della femminista Anna Maria Mozzoni pubblicato su "La Lega della democrazia" il 15 maggio 1880 in cui la Serao ribadisce come la donna in politica non debba avere opinioni ma sentimenti:

mi trovo costretta a dirle che quella certa specie di donna che è l'ideale delle sue teorie, è da noi assolutamente respinta, come una figura rigida, dura, ieratica, insugherita, senza alcuna bellezza, senza alcuna poesia. [...] noi la respingiamo come antiestetica. Per la vita, per l'amore, per l'arte ci vuole la donna. Istruita, ma donna. Maestra del popolo, infermiera, scrittrice, educatrice, ma donna. Niente diritti politici, niente ingerenze elettorali, niente attribuzioni maschili, niente professioni impossibili. [...] Sebbene a lei possa parere impossibile, ho letta la storia. Salvo le debite e nobili eccezioni, so di donne più o meno eroine, dalle virtù più $\mathrm{o}$ meno equivoche, dalle reputazioni più o meno usurpate. È il caso di chiedersi se queste donne avevano uno sposo, se lo amavano, se avevano dei figli, se sapevano insegnar loro l'alfabeto, se i mobili delle loro case erano spolverati a dovere, se sapevano cucire, rammendare, far di conti - cose molto importanti. [...] Io mi propongo di combattere in tutti i modi, ad oltranza, senza remissione [...] la falsa emancipazione, i diritti politici inutili e dannosi, la letteratura ampollosamente emancipatrice, i giornaletti femminili, clandestini ed emancipatori, la donnaapostolo, la donna-missione, la donna-principio (Buzzi, 1981: 88)

Anche la Bernardini riconosce che la donna ha un ruolo all'interno della società non per se stessa ma perché inglobata nello status familiare, cardine dell'ordinamento borghese e liberale del tempo. Tuttavia, con le sue opere mostra la profonda crisi di tale istituzione e la sempre maggiore coscienza che stavano prendendo le donne in relazione a questa più "moderna" ottica della loro collocazione all'interno della famiglia e della società. In tal senso si consuma ancor di più in divario con la rassegnata e prudente visione della Serao.

Le storie della Bernardini sono una rassegna "di donne sopraffatte da passioni inarginabili e nondimeno eticamente incolpevoli: non più eroine del male ma donne 
timide e ardite che espandono la loro potenzialità amorosa, fiancheggiate dalla complicità dell'autrice" che ne legittima i cedimenti "in nome di una simpatetica coscienza della comune condizione femminile" (Verdirame, 2009: 55-6). La scrittrice di Narni appare dunque una donna il cui buonsenso, nell'Ottocento postpositivistico e sullo sfondo dell'affaristica Italia umbertina, ha "dissolto la totalizzante chimera dell'amore romantico" incidendo sull'eros con una "scenografia semplice e calcolata" ove campeggiano figure "incostanti e falsamente innocenti, adattative, solo apparentemente trascinate dai sentimenti" (Verdirame, 2009: 56).

E certamente nella poetica della Bernardini non poté non influire l'ambiente in cui ha vissuto, quello della Catania di fine '800, e di un'intellighenzia con la quale, direttamente o indirettamente, era in costante colloquio grazie ai rapporti tenuti, non soltanto a livello nazionale, dal marito Luigi Capuana.

In un articolo commemorativo, apparso su "Il Popolo di Sicilia" il 20 marzo 1920, Adelide traccia una sorta di biografia della coppia Capuana Bernardini e, ricordando gli anni vissuti nella capitale, racconta:

I nostri due salotti romani pieni di sole, davano sulla piazza dell'Indipendenza così bellamente circondata da sontuosi villini e da filari di paulonia. Erano di tanto in tanto frequentati da scrittrici come Grazia Deledda, Matilde Serao, Mantea e da vecchi amici di Capuana, quali Ferdinando Martini, Giorgio Arcoleo e De Amicis, Verga, D'Annunzio quando capitavano a Roma; e da giovane amici quali Guelfo Civinini, Diego Angeli, Antonio Beltramelli ed altri molti. (Verdirame, 2009: 40)

Anche Catania, dove i coniugi fanno ritorno nel 1902, è fucina di una svariata messe di artisti che avrebbero segnato la storia della letteratura, dell'arte, del teatro e del cinema. È la Catania in cui si è ormai stabilito Giovanni Verga, dove operano Mario Rapisardi, Federico De Roberto, Nino Martoglio, Giovanni Grasso, Angelo Musco, ed altri.

Un ambiente fecondo e stimolante, dunque, anche per la letteratura femminile che porta avanti la propria ricerca alla scoperta di soggetti nuovi e di un punto di vista diverso, "esempio di modernità, e nello stesso tempo del permanere di alcuni topoi" nella rappresentazione della condizione delle donne. E, in tal senso, la Bernardini "ha puntato la lente sottile della critica sulle nuove istanze realistiche che la cultura europea ha proposto, allargando la sfera dei suoi interessi a temi e problemi di viva attualità, che hanno toccato direttamente la realtà conflittuale della società postunitaria, lacerata da tensioni ideologiche e morali che hanno investito, alla radice, gli istituti sui quali si è fondato il fragile tessuto sociale" (Licciadri, 2006) ${ }^{5}$.

5 Santoro nelle riedizione di L'altro dissidio, composta nel 1891, osserva che in esso vi sono contenuti tutti i temi della coeva questione femminile: il dolore per un amore non vissuto, l'adulterio, la presa di coscienza dell'identità femminile. 
Bisogna evidenziare la continuità dell'impegno letterario e da impresaria mostrata dalla Bernardini, già dall'indomani della morte del marito nel 1915, occupandosi di letteratura sino al 1931. Dopo un periodo di smarrimento, di sofferenza e di isolamento, infatti, Adelaide continua la sua opera di scrittrice e segue da vicino la promozione delle opere sue e di Capuana. Anche in questo caso non mancarono dissapori, forti dissidi, in specie con Musco e Martoglio, e mosse avventate, come quando la Bernardini distrugge le "riservatissime" lettere in cui Verga parlava all'amico Capuana di due suoi amori, Giselda Fojanesi e Donna Vittoria Cima (Zappulla Muscarà, 1987: 27-33)6 ; o come quando mette a repentaglio la reperibilità di preziosi autografi, fra cui quello de I Malavoglia, o polemizza aspramente con Pirandello reo di avere copiato dalla novella capuanaiana Dal taccuino di Ada per la redazione del I atto di Vestire gli ignudi (Sipala, 1974) ${ }^{7}$.

Sul fronte teatrale, l'impegno più significativo della Bernardini s'inserisce all'interno dell'avventura drammaturgica dialettale condotta da Luigi Capuana, a sua volta sviluppatasi nel complesso contesto storico-culturale del primo '900 che vede sorgere, quasi in sordina per poi deflagrare con inaspettato successo sia in Italia che all'estero, un teatro siciliano già adulto, portato alla ribalta da talenti straordinari quali Giovanni Grasso e Angelo Musco.

Dapprima "ostile al teatro dialettale, ritenuto una forma d'arte inferiore per mezzi, contenuto e intenti artistici, elemento di separatismo culturale, conformemente al clima post-unitario e alla giovanile aspirazione ad un teatro e ad una lingua nazionali", Capuana intorno al 1902, "rivisitando tesi pregiudiziali, comuni del resto molti intellettuali del tempo (si pensi a Verga e a Pirandello), smentite dalle vicende individuali e storiche, si lanciava nella nuova avventura dialettale con l'entusiasmo che lo caratterizzava". Un'attività che dipanandosi nell'arco di oltre un decennio e coprendo l'estrema stagione "lo doveva assorbire fino agli ultimi giorni della sua esistenza e a torto ritenuta marginale o quasi fase crepuscolare di un autore intellettualmente stanco che in essa si impegnava solo per sopperire alle pressanti angustie economiche" (Zappulla Muscarà, 1996: 551).

L'interesse per il dialetto e la volontà di farsi rappresentare da famosi artisti, unitamente alle sempre presenti urgenze economiche, spingono Capuana a tradurre (o come preferiva dire lui a "ridurre") numerose opere in vernacolo. Tra queste, i testi della moglie: Ammatula!, Di cosa nasci cosa, Sangu bonu non menti, La so' murali. E se

6 Distruggendole... ho creduto, anzi, ho sentito di fare la cosa giusta; ho sentito che le Care Ombre mi approvavano" scrive in una lettera a De Roberto il 3 giugno 1922.

7 Bernardini si servì del "Giornale d'Italia" del 21 e 22 novembre 1922 per esprimere in suo sdegno: "Capuana non è qui $[\ldots]$; ma $[. .$.$] qualche volta fa comodo plagiare i morti e passare sul$ cuore dei vivi $[\ldots]$. Ora lei fecondo commediografo com'è, dovrebbe buttare giù due altre commedie. "Spogliare i morti" e "Calunniare i vivi". 
l'adattamento scenico di Lu neu di Giovanni Meli, interrotto dalla morte del mineolo, voleva essere portato a termine proprio dalla Bernardini che però alla fine rinuncia, lei traspone invece in italiano le commedie del marito Il Cavalier Pedagna e Quacquarà, inserendo in quest' ultima (apparsa su "Nuova Antologia" ${ }^{8}$ ), nell'edizione postuma del 1921, un lieto fine non previsto dall'autore (Zappulla Muscarà, 1996: 554). La commedia è data a Musco che però non apprezza il finale. L'autografo di Capuana, difatti, reca in calce un "N.B." di mano della Bernardini che, astiosamente, annota: "La mezza pagina che manca fu spedita da Angelo Musco, per provargli che i tre atti di Quacquarà erano stati compiuti dall'autore; e questa umile verità è, ormai, nota a tutti. Ma Angelo Musco, e colui che vuol essere solo nel repertorio della "Compagnia Comica Siciliana" si affannano ancora a far credere e dire che il terzo atto di "Quacquarà" è opera mia! Vigliacchi! Ingrati!... Ada Capuana" (Zappulla Muscarà, 1996: 569).

Oltre che per Musco, gli strali sono anche per Martoglio già in precedenza oggetto di parole "dure" da parte della vedova di Capuana: "mi usi la cortesia di dire al suo compare Musco che da più di un mese gli ho spedito il copione di "Quacquarà" [...]. Egli non $\mathrm{mi}$ ha fatto sapere nulla di nulla. È anche questo villano silenzio...una rosa deposta su la tomba di Luigi Capuana?" (lettera del 4 febbraio 1916). A sua volta Martoglio, il 24 dicembre 1915, aveva scritto a Musco: “Io non lascerò, come Capuana, una donna viziosa e pettegola bisognosa di soddisfare la sua ambizione e i suoi vizi" prevalere (Zappulla Muscarà, 1996: 696).

Ed in effetti il rapporto con Martoglio è particolarmente tormentato e conflittuale, reso ancora più aspro dal carattere ombroso del belpassesse, anch'egli pronto alla lite e alla polemica.

Dopo la morte di Capuana, avvenuta a Catania, 29 novembre 2015 per paralisi cardiaca, la Bernardini che, con giovanile baldanza, ne aveva condiviso le difficoltà economiche e artistiche (diritti di autore, opere messe in scena in ritardo o alterate o perfino rifiutate, lavori letterari realizzati forzatamente), si dedica con determinazione alla salvaguardia ed alla gestione dell'opera del marito e, specialmente in ambito teatrale, ne sollecita spesso la messa in scena o la replica dei tanti lavori. In tale contesto, se montava il risentimento verso Pirandello, Verga e De Roberto, davvero incontenibile è l'astio nei confronti di Musco e, ancor più, di Martoglio che sovente ne era il portavoce.

In una lettera del 21 gennaio 1919 in cui il poliedrico artista la accusa di "volgare denigrazione" la Bernardini risponde: "Martoglio lei ha dato un nome troppo benigno al sentimento che per tre anni ho provato per Lei!... Non si tratta di volgare denigrazione: si tratta di odio, di odio rabbioso, anzi che è peggio. [...] Ma dopo aver saputo che Lei impose ad Angelo Musco di mettere in seconda linea le commedie di Capuana; dopo

$8 \quad 1^{\circ}$ settembre- 16 settembre- $1^{\circ}$ ottobre 1920. 
aver saputo che lei proibì allo stesso Capocomico di dare al Morgana un mio atto unico [...] dopo aver saputo che Lei aveva impedito che si svolgesse una Commemorazione; e, infine, dopo aver saputo tant'altre cose... il meno che potessi fare era di odiare Nino Martoglio e qualche altra persona!" (Zappulla Muscarà, 1996: 703).

Musco, da parte sua, è colpevole agli occhi della Bernardini di avere alterato alcune opere di Capuana per ottenete il consenso del pubblico a scapito dell'arte (si pensi allo "scandalo"9 suscitato da Lu Paraninfu, messo in scena nel 1916 che consacra Musco al successo tanto da divenirne il cavallo di battaglia) e, soprattutto, di dare troppo spazio alla sua amante Francesca Sabato Agnetta. In una lettera del 12 settembre 1915 di Capuana a Musco, così Ada saluta l'attore: "Anch'io la saluto tanto, ma... un po' a denti stretti, perché Lei non mi rappresenta mai, o quasi mai!"; in un'altra è lo stesso Capuana che si lamenta con Martoglio chiedendogli i copioni della moglie "che il Musco tiene in repertorio soltanto per burlarsi della Autrice" (Zappulla Muscarà, 1996: $667 ; 687)$.

Neanche la critica è stata benevola con la drammaturga. Hanno avuto recensioni negative Bufera ${ }^{10}$, Nonostante ${ }^{11}$, La so morali ${ }^{12}$; I bestia le viene rimandata indietro da Musco con questa motivazione: "Soggetto troppo vecchio pel mio nuovo repertorio d'Arte" (Zappulla Muscarà, 1996: 584; 623; 727).

Meno duri, invece, i giudizi su Fulvia Tei ${ }^{13}$, Di cosa nasci $\cos a^{14}$ e su Ammatula!, atto unico in cui si riprende la tematica napoletana di Assunta Spina di Salvatore Di Giacomo

9 Domenico Oliva in merito agli eccessi dell'attore, si chiede su "L'Idea Nazionale" del 26 gennaio 1916, se Capuana avrebbe approvato Musco "suo interprete, quando nel rappresentare gli effetti della paura, non rinunziò all'ultimo effetto fisico, sul quale la decenza mi vieta insistere, e che d'altronde, riprodotto con realismo integrale, pareva facesse crollare il teatro per risa inaudite".

10 Ruberti sul "Giornale d'Italia" del 13 agosto 1906 dichiara: “Non se l'abbia per male, l'egregia signora, ma questa sua Bufera rappresenta un errore facilmente perdonabile, del resto, perché breve. [...] Quello che nella penna della gentile scrittrice avrebbe prestato materia ad una indovinata novella, nella condotta scenica fa desiderare un maggior ossequio alle leggi della tecnica, accompagnato da una chiara nozione del carattere delle persone".

11 Sul "Giornale d'Italia" del 13 agosto 1910, F. Rain scrive: "Ben lontana dalla insipida banalità chè sostanza di troppe cose della produzione... minima d'oggi - la commedia applaudita iersera ha pure qualche difetto come, ad esempio, la prolissità dell'ultima scena e la poca originalità di qualche carattere".

12 Lo dico subito che non mi scappi via: siamo nel teatro d'eccezione, cerebrale o cervellotico, paradossale, o peggio assurdo. Persino in qualche spiccato atteggiamento ed in qualche motivazione che dovrebbe avere la virtù di essere nuova se non fosse scurrile, ricorda l'ultima maniera dell'illustre Luigi Pirandello. [...] Questo lavoro suona offesa, insulto alla Sicilia nostra. Ci si fa la figura degli imbecilli inconsapevoli", tuona sul "Corriere di Catania" del 20 settembre 1919 Carlo Condorelli.

13 Capuana scrivendo sia a De Roberto (4 agosto 1898) sia a Verga (22 agosto 1898) dichiara che "il drammettino in un atto" Fulvia Tei ha meravigliato tanto lui quanto Boutet.

14 Sul "Corriere di Catania" del 13 agosto 1914 si annuncia l'uscita della "spigliata commediola" interpretata da Musco: "un nuovo gioiello", anche se "troppa finezza, troppa miniatura sono profuse nel delicato lavoretto, sì che al nostro pubblico riuscirà difficile capire tutta la sensibilità che la gentildonna vi ha profuso". Messa in scena nell'aprile 1915, il recensore del "Corriere della sera" parla di "comicità garbata dell'atto" anche se è nella struttura "un po' ingenuo e un po' forzato". 
e di Rosa Esposito di Menotti Bianchi, caldeggiato fortemente da Capuana a Martoglio e successivamente venduto a Musco.

Alla luce di tutto ciò è oggi più che mai necessaria una ricognizione puntuale ed un serio ripensamento della figura e dell'opera della Bernardini, donna che, volente o nolente, è stata una protagonista sia in ambito letterario che imprenditoriale nell' intenso e cruciale periodo della fine dell'800- metà del '900 in Sicilia, luogo cardine in cui si muovevano autori di primo piano ed emergevano generi ed esperienze tra i più significativi della letteratura contemporanea.

\section{REFERENCIAS Y BIBLIOGRAFÍA}

Bandini Buti, M., Poetesse e scrittrici, Roma, Istituto Editoriale Romano, 1946, Licciardi, G.- Santoro, A., Adelaide Bernardini, in Siciliane. Dizionario biografico, a cura di M. Fiume, Siracusa, E. Romeo, 2006.

Marchese, D., La Penna e l'ago. Matilde Serao: la scrittrice, la donna, X Congreso Internacional del Grupo de Investigación Escritoras e Escrituras, Ausencias. Escritoras en los márgenes de la cultura (Madrid, 24-26 octubre 2013), editoras Mercedes Arriaga Flòrez, Salvatore Bartolotta, Milagro Martín Clavijo, pp. 754- 765.

Mazzamuto, P., Luigi Capuana. Teatro dialettale siciliano, Catania, Giannotta, 1974.

Sipala, P.M., Capuana e Pirandello. Storia e testi di una relazione letteraria, Catania, Bonanno, 1974.

Verdirame, R., Narratrici e lettrici (1850-1950). Le letture della nonna dalla Contessa Lara a Luciana Peverelli, Padova, Libreria Universitaria, 2009.

Verdirame, R., Polemiche e "bagattelle" letterarie tra Otto e Novecento, Catania, CUECM, 2009, pp. 33-60.

Verdirame, R., “Una letterata pseudomodernista: Adelaide Bernardini Capuana e una sua novella dispersa", Le forme e la storia, n.s. III, 1 (2010), pp. 265-276.

Zappulla Muscarà, S., Capuana e De Roberto, Caltanissetta-Roma, 1987.

Zappulla Muscarà, S., Luigi Capuana e le carte messaggiere, Catania, CUECM, 1996. 